\title{
The Demonic Product of Probabilistic Relations
}

\author{
Ernst-Erich Doberkat \\ Chair for Software Technology \\ University of Dortmund \\ doberkat@acm.org
}

\begin{abstract}
The demonic product of two probabilistic relations is defined and investigated. It is shown that the product is stable under bisimulations when the mediating object is probabilistic, and that under some mild conditions the non-deterministic fringe of the probabilistic relations behaves properly: the fringe of the product equals the demonic product of the fringes.
\end{abstract}

Keywords: Probabilistic relations, bisimulation, demonic product, concurrency.

\section{Introduction}

Let $R \subseteq A \times B$ and $S \subseteq B \times C$ be set theoretic relations, then, interpreting $R$ and $S$ as filters which form the pipe $R \circ S$, input a may yield output $c$ iff there exists an intermediate value $b$ such that both $\langle a, b\rangle \in R$ and $\langle b, c\rangle \in S$ hold, hence input $a$ produces output $b$ via $R$ which in turn is fed into $S$ giving $c$. This is an angelic version of the composition of two relations. Demonic behavior, however, is worst case behavior: if something bad can happen, it happens. Thus if on input $a$ relation $R$ produces an output $b$ which is not in the domain of relation $S$, then $a$ will not be in the domain of the demonic product of $R$ and $S$. This consideration leads [5] p. 169] to the definition of the demonic product

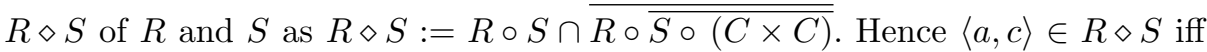
$\langle a, c\rangle \in R \circ S$, and if for all $b \in B$ the following holds: if $\langle a, b\rangle \in R$, then there exists $c^{\prime} \in C$ such that $\left\langle b, c^{\prime}\right\rangle \in S$.

When systems are modelled as stochastic systems rather than non-deterministic ones, demonic behavior can still be described as worst case behavior. In the discrete case we would say that $c$ is the possible output of the demonic product of two probabilistic relations $K$ and $L$ upon input $a$ iff $c$ may be produced by the usual product of $K$ and $L$ with positive probability, and if the following holds: whenever $K(a)(b)>0$ through a terminating computation, we can always find $c^{\prime} \in C$ such that $L(b)\left(c^{\prime}\right)>0$, and the computation for $L$ terminates upon input $b$. Widening the scope to probabilities on non-countable spaces, this description has to be adjusted somewhat, because positive probabilities may not necessarily be assigned to single outputs. This is what the present paper is about: we show how to carry over the definition of demonic product from set theoretic relations to probabilistic ones, and we investigate this product.

M. Nielsen and U. Engberg (Eds.): Fossacs 2002, LNCS 2303, pp. 114-128 2002.

(C) Springer-Verlag Berlin Heidelberg 2002 
Probabilistic relations are the stochastic counterparts to set based relations (see [1 16] or [7]). A probabilistic relation is a transition kernel between two measurable spaces, combining measurable maps with subprobability measures (a formal definition is given in Sect. 2). We employ subprobability measures here rather than their probabilistic step-twins because they permit modelling non-terminating computations: if $K(x)(B)$ is the probability that $K$ with input $x \in X$ produces an output which lies in $B \subseteq Y$, then $K(x)(Y)<1$ means that the computation does not produce an output with probability 1 , i.e., that the computation does not necessarily terminate. The demon, however, forces us to continue only on inputs that guarantee termination, because only then the next computation $L$ may start with an input that $K$ has produced with certainty. This then leads to the probabilistic version $K \star L$ of the demonic product of $K$ and $L$.

The analogy between set theoretic and probabilistic relations flows from two sources. First and informally, set theoretic relations may be used for nondeterministic processes, so that $\{y \mid\langle x, y\rangle \in R\}$ yields the set of all possible results of computation $R$ after input $x$. Many applications, however, assign probabilities to possible outcomes (because some events carry more weight than others), and this leads to the notion of a transition probability which gives us the probability $K(x)(B)$ that upon input $x$ the output will be an element of set $B$. The intuitive reasoning is supported formally, as e.g. [16] points out: the power set functor forms a monad in the category of sets and has set theoretic relations as the Kleisli construction; the functor assigning each measurable space its probability measures forms also a monad and has Markov kernels as its Kleisli construction, see [10]. In this way a categorical underpinning of the intuitive reasoning is provided.

This paper discusses the demonic product of two transition kernels, or probabilistic relations, as we will also call them, it proposes a definition for this product, and it investigates two properties, viz., stability under bisimulation and the behavior of an associated set theoretic relation. Two notions of bisimulations 4,18] are defined for transition kernels, and it is shown that they are very closely related. Then we show that bisimililarity is preserved through the ordinary, and through the demonic product: if the factors are bisimilar, and if the bisimulation is related through a probabilistic object, so are the respective products. For each probabilistic relation $K$ we can find a set theoretic relation supp $K$ that characterizes the unweighed outcome of $K$. This correspondence was investigated in depth in [6] with methods originating from stochastic dynamic programming, it is strengthened here to a relation supp* $K$ (called the non-deterministic fringe of $K$ ) by taking termination into account. It is shown that under some mild conditions calculating the demonic product and forming the set theoretic relation may be interchanged, so that supp* $(K \star L)=\operatorname{supp}^{*} K \diamond \operatorname{supp}^{*} L$ holds.

Most of this is only possible under some topological assumptions that are satisfied e.g. when considering the real line. Some preparations are needed, they are provided in Sect. 2. Sect. 3 defines the demonic product and investigates some basic properties like associativity, Sect. 4 suggests two definitions of bisim- 
ilarity, relates them to each other, selects one for further work, and shows that bisimilarity and forming the product are compatible. Sect. 5 compares the set theoretic and the probabilistic demonic products, and Sect. 6] offers some conclusions together with some suggestions for further work. Most proofs had to be omitted for reasons of space; the reader is referred to the technical report [8] in which all proofs are provided.

Acknowledgements. Part of this work was done while I was visiting what is now the Dipartimento di Informatica at the University of L'Aquila. This research was in part supported through grants from the Exchange Programme for Scientists between Italy and Germany from the Italian Minstry of Foreign Affairs/Deutscher Akademischer Austauschdienst and from Progetto speciale I.N.D.A.M./GNIM "Nuovi paradigmi di calcolo: Linguaggi e Modelli". Professor Fabio Spizzichino made it possible for me to work in the impressive and exciting Mathematics Library of La Sapienza in Rome. I want to thank him, and Professor Eugenio Omodeo, my host in L'Aquila. The referees' comments are appreciated.

\section{Preliminaries}

This section provides for the reader's convenience some notions from measure theory and topology. We introduce transition kernels as, the probabilistic counterpart of set theoretic relations, as members of a suitably chosen comma category, remind the reader of Polish spaces, and give some basic definitions for relations.

Transition Kernels. Let $\mathcal{M}$ be the category of all measurable spaces with measurable maps as morphisms. Denote by $\boldsymbol{S}(X)$ the set of all subprobability measures on (the $\sigma$-algebra of) $X$; we usually omit the $\sigma$-algebra from the notation, when the context is clear, and talk about measurable subsets as the members of it. $\boldsymbol{S}(X)$ is endowed with the $*-\sigma$-algebra, i.e. the smallest $\sigma$-algebra that makes for each measurable subset $A$ of $X$ the map $\mu \mapsto \mu(A)$ measurable. $P(X)$ consists of all probability measures on $X$.

Put for $f: X \rightarrow Y$ and $\mu \in \boldsymbol{S}(X) \boldsymbol{S}(f)(\mu): B \mapsto \mu\left(f^{-1}[B]\right)$, (the image of $\mu$ under $f ; \boldsymbol{P}(f)$ is defined through exactly the same expression, having $\boldsymbol{P}(X)$ and $\boldsymbol{P}(Y)$ as domain, and as range, resp.), then $\boldsymbol{S}(f) \in \boldsymbol{S}(Y)$. Consequently, $\boldsymbol{S}$ and $\boldsymbol{P}$ are functors $\mathcal{M} \rightarrow \mathcal{M}$, because $\boldsymbol{S}(f): \boldsymbol{S}(X) \rightarrow \boldsymbol{S}(Y)$ and $\boldsymbol{P}(f)$ : $\boldsymbol{P}(X) \rightarrow \boldsymbol{P}(Y)$ are $*-\sigma$-measurable whenever $f: X \rightarrow Y$ is measurable. The functor $\boldsymbol{P}$ has been investigated by Giry $[10$.

We will work in the comma category $\mathbb{1}_{\mathcal{M}} \downarrow S$ [12, II.6] which has as objects triplets $\langle X, Y, K\rangle$ with objects $X, Y$ from $\mathcal{M}$ and a morphism $K: X \rightarrow \boldsymbol{S}(Y)$ $\left(\mathbb{1}_{\mathcal{M}}\right.$ is the identity functor on $\left.\mathcal{M}\right)$. Thus $K$ has the following properties:

1. $x \mapsto K(x)(B)$ is a measurable map for each measurable subset $B$ of $Y$,

2. $K(x) \in \boldsymbol{S}(Y)$ for each $x \in X$, thus $K(x)$ is a measure on the $\sigma$-algebra of $Y$ such that $K(x)(Y) \leq 1$ holds. 
Hence $K$ is a transition kernel from $X$ to $Y$ in the parlance of probability theory. If $K(x)(Y)=1$ holds for each $x \in X$, then $K$ is called a transition probability or a Markov kernel, and the corresponding object $\langle X, Y, K\rangle$ a probabilistic object. In what follows, $K: X \stackrel{\bullet}{\hookrightarrow} Y$ indicates that $K$ is a transition kernel from $X$ to $Y$. These kernels are also called probabilistic relations [1/7 16].

Given $\mu_{i} \in \boldsymbol{S}\left(X_{i}\right)$, the product measure $\mu_{1} \otimes \mu_{2}$ assigns to measurable rectangles $A_{1} \times A_{2}$ the product of the single measures: $\left(\mu_{1} \otimes \mu_{2}\right)\left(A_{1} \times A_{2}\right):=$ $\mu_{1}\left(A_{1}\right) \cdot \mu_{2}\left(A_{2}\right)$. The product is uniquely determined by this property, since the set of all measurable rectangles generates the product $\sigma$-algebra and is closed under finite intersections; whenever we talk about the product of measurable spaces, we assume that the $\sigma$-algebra on the Cartesian product is the smallest $\sigma$-algebra that contains all rectangles. The product measure has the following property: if $f: X_{1} \times X_{2} \rightarrow \boldsymbol{R}$ is measurable and bounded, then

$$
\begin{aligned}
\int_{X_{1} \times X_{2}} f d \mu_{1} \otimes \mu_{2} & =\int_{X_{2}} \int_{X_{1}} f\left(x_{1}, x_{2}\right) \mu_{1}\left(d x_{1}\right) \mu_{2}\left(d x_{2}\right) \\
& =\int_{X_{1}} \int_{X_{2}} f\left(x_{1}, x_{2}\right) \mu_{2}\left(d x_{2}\right) \mu_{1}\left(d x_{1}\right)
\end{aligned}
$$

by Fubini's Theorem. Now let $\mu \in \boldsymbol{S}(X)$ and $K: X \stackrel{\bullet}{\hookrightarrow} Y$, then

$$
(\mu \otimes K)(A):=\int_{X} K(x)\left(A_{x}\right) \mu(d x)
$$

defines a measure on the product $X \times Y$ (where $A_{x}:=\{y \mid\langle x, y\rangle \in A\}$ ). If $f: X \times Y \rightarrow \boldsymbol{R}$ is measurable and bounded, then

$$
\int_{X \times Y} f d \mu \otimes K=\int_{X} \int_{Y} f(x, y) K(x)(d y) \mu(d x) .
$$

A morphism between $\langle X, Y, K\rangle$ and $\left\langle X^{\prime}, Y^{\prime}, K^{\prime}\right\rangle$ in $\mathbb{1}_{\mathcal{M}} \downarrow \boldsymbol{S}$ is a pair $\langle f, g\rangle$ of measurable maps $f: X \rightarrow X^{\prime}, g: Y \rightarrow Y^{\prime}$ for which $K^{\prime} \circ f=\boldsymbol{S}(g) \circ K$ holds. Hence

$$
K^{\prime}(f(x))(B)=\boldsymbol{S}(g)(K(x))(B)=K(x)\left(g^{-1}[B]\right)
$$

is true for $x \in X$ and the measurable set $B \subseteq Y^{\prime}$.

Denote finally the indicator function $\chi_{A}$ of a set $A$ by

$$
\chi_{A}(x):=(x \in A ? 1: 0) .
$$

Polish Spaces. A Polish space is a second countable topological space which is metrizable with a complete metric. Polish spaces have always their Borel sets as their measurable structure, hence we talk also about Borel sets when addressing measurable subsets of a topological space. The Polish space $X$ induces a metric structure on $\boldsymbol{S}(X)$ through the topology of weak convergence which is characterized through the famous Portmanteau Theorem [17]:

Proposition 1. The following conditions are equivalent for a sequence $\left(\mu_{n}\right)_{n \geq 0}$ of finite measures and for the finite measure $\mu$ on the Polish space $X$ : 
1. $\mu_{n}$ converges weakly to $\mu$,

2. $\int_{X} f d \mu_{n} \rightarrow \int_{X} f d \mu$ for each bounded and continuous $f: X \rightarrow \boldsymbol{R}$,

3. $\liminf _{n \rightarrow \infty} \mu_{n}(F) \leq \mu(F)$ for each closed subset $F \subseteq X$.

With this topology, $\boldsymbol{S}(X)$ becomes a Polish space with $\boldsymbol{P}(X)$ as a closed subspace. The $*-\sigma$-algebra are the Borel sets for the topology of weak convergence.

The Borel sets on a Polish space exhibit a certain flexibility because they do not uniquely determine the topology; in fact, the topology may be manipulated a bit without affecting the Borel structure. We will make use of this rather surprising fact, which is quoted from [19, Corollary 3.2.6]:

Proposition 2. Suppose $(X, \mathcal{T})$ is a Polish space, $Y$ a separable metric space, and $f: X \rightarrow Y$ a measurable map. Then there is a finer Polish topology $\mathcal{T}^{\prime}$ on $X$ generating the same Borel $\sigma$-algebra such that $f:\left(X, \mathcal{T}^{\prime}\right) \rightarrow Y$ is continuous.

Define for $\mu \in \boldsymbol{S}(X)$ (X Polish) the $\operatorname{support} \operatorname{supp}(\mu)$ of $\mu$ as the smallest closed subset $F \subseteq X$ such that $\mu(F)=\mu(X)>0$, hence for positive $\mu(X)$

$$
\operatorname{supp}(\mu)=\bigcap\{F \mid F \subseteq X \text { is closed, } \mu(F)=\mu(X)\}
$$

Because finite measures on Polish spaces are $\tau$-additive, $\mu(\operatorname{supp}(\mu))=\mu(X)$, and $x \in \operatorname{supp}(\mu)$ iff $\mu(U)>0$ for each neighborhood $U$ of $x$. The support for the null measure is defined as the empty set.

Relations. A relation $R$ is, as usual, a subset of the Cartesian product $X \times Y$ of two sets $X$ and $Y$. The universal relation on $X$ is just $U_{X}:=\{\langle x, y\rangle \mid x, y \in X\}$, and sometimes we will need a part of the diagonal $\Delta_{A}:=\{\langle x, x\rangle \mid x \in A\}$.

Assume that $X$ carries a measurable structure, and that $Y$ is a Polish space. If for each $x \in X$ the set valued map induced by $R$ (and again denoted by $R$ )

$$
R(x):=\{y \in Y \mid\langle x, y\rangle \in R\}
$$

takes closed and non-empty values, and if the weak inverse

$$
(\exists R)(G):=\{x \in X \mid R(x) \cap G \neq \emptyset\}
$$

is a measurable set, whenever $G \subseteq Y$ is open, then $R$ is called a measurable relation. Since $Y$ is Polish, $R$ is a measurable relation iff the strong inverse

$$
(\forall R)(F):=\{x \in X \mid F(x) \subseteq F\}
$$

is measurable, whenever $F \subseteq Y$ is closed [11, Theorem 3.5].

Transition kernels yield measurable relations in a natural way: $K: X \stackrel{\bullet}{\hookrightarrow} Y$ with $K \neq 0$ induces a relation

$$
\text { supp } K:=\{\langle x, y\rangle \mid x \in X, y \in \operatorname{supp}(K(x))\}
$$


so that $\operatorname{supp}(K(x))$ takes closed values, and it is measurable, because for an open set $G \subseteq Y$ the weak inverse

$$
(\exists \operatorname{supp} K)(G)=\{x \in X \mid K(x)(G)>0\}
$$

is measurable. Measurability is also easily established for the strong inverse $(\forall$ supp $K)(F)$ whenever $F \subseteq Y$ is closed, and, somewhat surprisingly, $F$ may be replaced by an arbitrary measurable subset $A \subseteq Y$, as we will see in Prop. 3 . This seems to be a peculiar property of set valued maps induced by the support of transition kernels; the general situation is that the weak inverse of a general measurable subset if analytic [11, Theorem 3.5].

\section{The Demonic Product}

For motivating the demonic product of probabilistic relations, we first have a look at the situation in the set based case. This then leads in quite a natural way to the definition for the probabilistic case. We need some preparations for the definition proper, mainly showing that sets interesting us here are measurable. As a byproduct we can establish the measurability of the strong inverse of a measurable set under a measurable relation, provided this relation is generated from a transition kernel. This is a rather surprising result when viewed from the theory of measurable relations. Some elementary properties for the demonic product are established, it is shown to be associative but not to posses the Dirac kernel as a neutral element.

Let $R \subseteq A \times B, S \subseteq B \times C$ be relations. The demonic product $R \diamond S$ of $R$ and $S$ is defined as

$$
R \diamond S:=R \circ S \cap \overline{R \circ \overline{S \circ U_{C}}} .
$$

Thus $\langle a, c\rangle \in R \diamond S$ iff $\langle a, c\rangle \in R \circ S$, and if it is not possible from $a$ by $R$ to reach a $b$ that is not in the domain of $S$ [5, p. 169], hence $\langle a, c\rangle \in R \diamond S$ iff these conditions are satisfied:

1. $\langle a, c\rangle \in R \circ S$,

2. $\forall b \in B:\left[\langle a, b\rangle \in R \Rightarrow \exists c^{\prime} \in C:\left\langle b, c^{\prime}\right\rangle \in S\right]$

Call for the moment $a \in A S$-extendable iff whenever $\langle a, b\rangle \in R$, then $\left\langle b, c^{\prime}\right\rangle \in S$ for some $c^{\prime} \in C$, hence $\langle a, c\rangle \in R \diamond S$ iff $\langle a, c\rangle \in R \circ S$, and $a$ is $S$-extendable.

Example 1. Put $X:=\{1,2,3\}, Y:=\{a, b, c\}$ and $Z:=\{\alpha, \beta, \gamma\}$, and let

$$
\begin{aligned}
R & :=\{\langle 2, a\rangle,\langle 2, b\rangle,\langle 2, c\rangle,\langle 3, a\rangle,\langle 3, c\rangle\}, \\
S & :=\{\langle a, \alpha\rangle,\langle a, \beta\rangle,\langle a, \gamma\rangle,\langle b, \alpha\rangle,\langle b, \beta\rangle,\langle b, \gamma\rangle\} .
\end{aligned}
$$

Then only 3 is $S$-extendable, and

$$
R \diamond S=\{\langle 3, \alpha\rangle,\langle 3, \beta\rangle,\langle 3, \gamma\rangle\}
$$


Now suppose that we have stochastic relations $K: X \stackrel{\bullet}{\hookrightarrow} Y$ and $L: Y \stackrel{\bullet}{\hookrightarrow}$ $Z$ for the Polish spaces $X, Y$ and $Z$. We want to model computations with these kernels. Intuitively, the $K$-computation terminates on input $x$ and yields output $y$ iff both $K(x)(Y)=1$ and $y \in \operatorname{supp}(K(x))$ are satisfied. Note that we usually cannot assign positive measure to any single point $y$, so $K(x)(\{y\})$ is not a suitable object for argumentation, which is the reason why we resort to $\operatorname{supp}(K(x))$ as manifesting the set of possible results. Termination of the computation for input $x$ is thus described by membership of $x$ in the set $\{x \in$ $X \mid K(x)(Y)=1\}$. The output of this $K$-computation is thus fed into $L$, and we postulate that then the $L$-computation terminates, too. Call by analogy $x \in X$ $L$-extendable iff the $K$-computation on input $x$ terminates with an output for which the $L$-computation also terminates, hence, iff the conditions

1. $K(x)(Y)=1$,

2. $\operatorname{supp}(K(x)) \subseteq \operatorname{Tr}(L)$

are both satisfied, where we have defined $\operatorname{Tr}(L):=\{y \in Y \mid L(y)(Z)=1\}$.

Hence a demon will permit the combined $(K, L)$-computation to terminate, and to produce a result on input $x$ iff the conditions above are satisfied for $x$. Then the result is produced in the usual manner.

Example 2. Continuing Example 1] define the transition kernels $K: X \stackrel{\bullet}{\hookrightarrow}$, and $L: Y \stackrel{\bullet}{\hookrightarrow} Z$ as follows:

\begin{tabular}{|c|c|c|}
\hline$K \mid a \quad b \quad c$ & & $L \mid \alpha \beta \gamma$ \\
\hline \begin{tabular}{l|lll}
1 & 0 & .3 & .4
\end{tabular} & ond & \begin{tabular}{l|l}
$a$ & 3.3 .4
\end{tabular} \\
\hline \begin{tabular}{l|lll}
2 & .25 & .25 & .5
\end{tabular} & and & \begin{tabular}{l|lll}
$b$ & .6 .1 .1
\end{tabular} \\
\hline \begin{tabular}{l|lll}
3 & .5 & 0 & .5
\end{tabular} & & $c \mid .2 .3 .5$ \\
\hline
\end{tabular}

Then $\operatorname{Tr}(K)=\{2,3\}, \operatorname{Tr}(L)=\{a, c\}$ and

$$
K(x)(Y)=1 \wedge \operatorname{supp}(K(x)) \subseteq \operatorname{Tr}(L) \Leftrightarrow x=3 .
$$

The following equalities are straightforward:

$$
\operatorname{supp}(K(1))=\{b, c\}, \operatorname{supp}(K(2))=\{a, b, c\}, \operatorname{supp}(K(3))=\{a, c\} .
$$

It is easy to see that 1 is not $L$-extendable, since $K(1)(Y)<1$, and that 2 is not $L$-extendable, because $\operatorname{supp}(K(2)) \nsubseteq \operatorname{Tr}(L)$ holds; input 3, however, turns out to be $L$-extendable.

The product $K ; L$ of the transition kernels $K$ and $L$ is defined as usual through $(K ; L)(x)(C):=\int_{Y} L(y)(C) K(x)(d y),(x \in X, C$ is a measurable subset of $Z$ ), and $K ; L: X \stackrel{\bullet}{\hookrightarrow} Z$ is easily established.

Before defining the demonic product for the probabilistic case, we need some technical preparations.

Proposition 3. Let $X$ be a measurable space, $Y$ and $Z$ Polish spaces with tran-

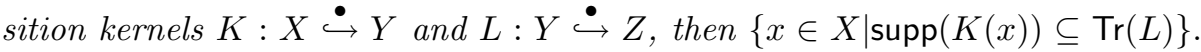
is a measurable subset of $X$. 
Note that the set $\operatorname{Tr}(L)$ introduced above is just the set of points on which $L$ is a probability measure; modelling computations using transition kernels, $\operatorname{Tr}(L)$ represents the set of terminating computations.

Prop. 3 has a surprising consequence for the strong inverse of general measurable sets under the measurable relation supp $K$ :

Corollary 1. Let under the assumptions of Proposition $3 \subseteq Y$ be a measurable subset. Then $(\forall$ supp $K)(A)$ is measurable.

Returning to the preparations for the definition of the demonic product, we observe that the expression $\operatorname{supp}(K(x)) \subseteq \operatorname{Tr}(L)$ is sometimes a little impractical to handle; it may be replaced by $K(x) \otimes L \in \boldsymbol{P}(Y \times Z)$, provided the input $x$ to $K$ makes $K$ 's computation terminate. Consequently, the former expression is equivalent for those inputs to $(K(x) \otimes L)(Y \times Z)=1$. Note that this equivalence depends critically upon Prop. 3 .

Corollary 2. Define under the assumptions of Proposition 3 the measurable sets

$$
\begin{aligned}
V(K, L) & :=\{x \in \operatorname{Tr}(K) \mid \operatorname{supp}(K(x)) \subseteq \operatorname{Tr}(L)\} \\
W(K, L) & :=\{x \in \operatorname{Tr}(K) \mid K(x) \otimes L \in \boldsymbol{P}(Y \times Z)\}
\end{aligned}
$$

Then $V(K, L)=W(K, L)$ holds.

Define the L-relativization $K_{L}^{\natural}$ of $K$ by $K_{L}^{\natural}(x):=\chi_{W(K, L)}(x) \cdot K(x)$. Consequently, $K_{L}^{\natural}$ assumes the value of $K(x)$ on $W(K, L)$, and shrinks to the null measure on the complement. Corollary 2 makes sure that $K_{L}^{\natural}: X \stackrel{\bullet}{\hookrightarrow} Y$ holds.

Definition 1. Let $X, Y$ and $Z$ be Polish spaces, and assume that $K: X \stackrel{\hookrightarrow}{\hookrightarrow}$ and $L: Y \stackrel{\bullet}{\hookrightarrow} Z$ are transition kernels. The demonic product $K \star L$ of $K$ and $L$ is defined as $K \star L:=K_{L}^{\natural} ; L$.

The following is immediate:

Observation 1 Under the assumptions of Definition [1] we have

1. $K \star L: X \stackrel{\bullet}{\hookrightarrow} Z$,

2. for each bounded and measurable $f: Z \rightarrow \boldsymbol{R}$

$$
\int_{Z} f d(K \star L)(x)=\chi_{W(K, L)}(x) \cdot \int_{Y}\left(\int_{Z} f(z) L(y)(d z)\right) K(x)(d y) \cdot \square
$$

Example 3. Define $K$ and $L$ as in Example 2, then

\begin{tabular}{c|lll}
$K ; L$ & $\alpha$ & $\beta$ & $\gamma$ \\
\hline 1 & .26 & .15 & .23 \\
2 & .325 & .25 & .375 \\
3 & .25 & .3 & .45
\end{tabular}

and

\begin{tabular}{c|lll}
$K \star L$ & $\alpha$ & $\beta$ & $\gamma$ \\
\hline 1 & 0 & 0 & 0 \\
2 & 0 & 0 & 0 \\
3 & .25 & .3 & .45
\end{tabular}

are easily established. 
Some elementary properties are collected in the next Proposition. Before stating and proving them, it will be helpful to calculate some $W$-sets. The next technical lemma states that termination of compound processes may be considered at different stages during their composition. The result for the entire process, however, is the same. We need this statement of course for establishing associativity of the demonic product in Prop. 4

Lemma 1. $W(K \star L, M)=W(K, L \star M)$.

Some elementary properties of the demonic product are collected for convenience, amplification and illustration. It turns out in particular that the demonic product $K \star L$ coincides with the usual one provided every input to $K$ leads to a terminating computation.

Proposition 4. Let $\boldsymbol{I}_{X}$ be the Dirac kernel on $X$, then

1. $\boldsymbol{I}_{X} \star K=\chi_{\operatorname{Tr}(K)} \cdot K$,

2. $K \star \boldsymbol{I}_{Y}=K_{\boldsymbol{I}_{Y}}^{\natural}$,

3. if $\operatorname{Tr}(K)=X$, then $K \star L=K ; L$,

4. the demonic product is associative.

\section{Bisimulations}

The similarity in the behavior of transition kernels is captured through bisimulations, which are introduced as span of morphisms:

Definition 2. Let $O_{1}$ and $O_{2}$ be objects in $\mathbb{1}_{\mathcal{M}} \downarrow \boldsymbol{S}$ with $O_{i}=\left\langle X_{i}, Y_{i}, K_{i}\right\rangle(i=$ 1, 2).

1. An object $P=\langle X, Y, K\rangle$ in $\mathbb{1}_{\mathcal{M}} \downarrow \boldsymbol{S}$ together with morphisms $\sigma_{1}=\left\langle s_{1}, t_{1}\right\rangle$ : $P \rightarrow O_{1}$ and $\sigma_{2}=\left\langle s_{2}, t_{2}\right\rangle: P \rightarrow O_{2}$ is called a 1-bisimulation for $O_{1}$ and $\mathrm{O}_{2}$.

2. If $X$ is a measurable subset of $X_{1} \times X_{2}$, and if both $\sigma$ and $\tau$ are constituted by the corresponding projections, then $P$ is called a 2-bisimulation for $O_{1}$ and $\mathrm{O}_{2}$. In this case $P$ will be written as $\langle X, K\rangle$.

$P$ may be interpreted as an object mediating between $O_{1}$ and $O_{2}$. A 1bisimulation makes the following diagram commutative:

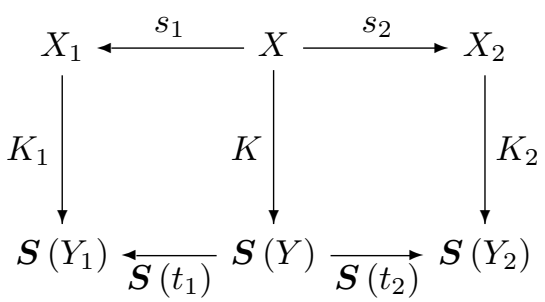

Suppose that $\mathcal{B}_{i}$ is the $\sigma$-algebra on $Y_{i}(i=1,2)$, then $\mathcal{B}_{0}:=g_{1}^{-1}\left[\mathcal{B}_{1}\right] \cap g_{2}^{-1}\left[\mathcal{B}_{2}\right]$ is the $\sigma$-algebra of all shared events on $Y$ (via $g_{1}, g_{2}$ ), so that $B \in \mathcal{B}_{0}$ iff we can 
find $B_{i} \in \mathcal{B}_{i}$ with $g_{1}^{-1}\left[B_{1}\right]=B=g_{2}^{-1}\left[B_{2}\right]$. The transition kernels $K_{1} \circ s_{1}$ and $K_{2} \circ s_{2}$ coincide on $\mathcal{B}_{0}$ :

$$
\begin{aligned}
K_{1}\left(s_{1}(x)\right)\left(B_{1}\right) & =K(x)\left(g_{1}^{-1}\left[B_{1}\right]\right) \\
& =K(x)(B) \\
& =K(x)\left(g_{2}^{-1}\left[B_{2}\right]\right) \\
& =K_{2}\left(s_{2}(x)\right)\left(B_{2}\right) .
\end{aligned}
$$

In this sense, bisimilar objects display the same behavior on shared events. In particular, if one of them terminates, the other will, too, since $Y \in \mathcal{B}_{0}$.

It is plain that there exists always a 2-bisimulation between probabilistic objects $O_{1}=\left\langle X_{1}, Y_{1}, K_{1}\right\rangle$ and $O_{2}=\left\langle X_{2}, Y_{2}, K_{2}\right\rangle$ : define the mediating object $P:=\left\langle X_{1} \times X_{2}, Y_{1} \times Y_{2}, K\right\rangle$, where $K\left(x_{1}, x_{2}\right):=K_{1}\left(x_{1}\right) \otimes K_{2}\left(x_{2}\right)$ is the product of $K\left(x_{1}\right)$ and $K\left(x_{2}\right)$. Then both $K_{1}\left(x_{1}\right)\left(A_{1}\right)=K\left(x_{1}, x_{2}\right)\left(A_{1} \times Y_{2}\right)$ and $K_{2}\left(x_{2}\right)\left(A_{2}\right)=K\left(x_{1}, x_{2}\right)\left(Y_{1} \times A_{2}\right)$ hold, whenever $A_{i} \subseteq Y_{i}$ is a measurable subset $(i=1,2)$. Consequently, the projections

$$
\begin{aligned}
& \left\langle\pi_{X_{1} \times X_{2}}^{X_{1}}, \pi_{Y_{1} \times Y_{2}}^{Y_{1}}\right\rangle: P \rightarrow O_{1} \\
& \left\langle\pi_{X_{2} \times X_{2}}^{X_{1}}, \pi_{Y_{2}}^{Y_{1} \times Y_{2}}\right\rangle: P \rightarrow O_{2}
\end{aligned}
$$

are the desired morphisms. Thus bisimulations are only non-trivial for the nonprobabilistic objects in $\mathbb{1}_{\mathcal{M}} \downarrow \boldsymbol{S}$, i.e. for such objects the termination of which cannot always be guaranteed.

The following observation shows why we may and do restrict our attention to 2-bisimulations:

Proposition 5. Let $O_{i}=\left\langle X_{i}, Y_{i}, K_{i}\right\rangle$ be objects in $\mathbb{1}_{\mathcal{M}} \downarrow \boldsymbol{S}$, then the following conditions are equivalent:

1. There exists a 1-bisimulation $\left\langle P,\left\langle s_{1}, t_{1}\right\rangle,\left\langle s_{2}, t_{2}\right\rangle\right\rangle$ with $P=\langle X, Y, K\rangle$ for $O_{1}$ and $O_{2}$ such that $\left\{\left\langle s_{1}(x), s_{2}(x)\right\rangle \mid x \in X\right\}$ is a measurable subset of $X_{1} \times X_{2}$.

2. There exists a 2-bisimulation for $\mathrm{O}_{1}$ and $\mathrm{O}_{2}$.

Let us comment briefly on the condition that $\left\{\left\langle s_{1}(x), s_{2}(x)\right\rangle \mid x \in X\right\}$ is a measurable subset of $X_{1} \times X_{2}$. This condition is in general not easy to handle, working in Polish spaces, however, a well-known Theorem attributed to ArseninKunugui [19, 5.12.1] renders it somewhat more practical:

Observation 2 Let $X, X_{1}, X_{2}$ be Polish spaces, and assume that $s_{i}: X \rightarrow X_{i}$ is measurable. Assume that $\left\{x \in X \mid\left\langle s_{1}(x), s_{2}(x)\right\rangle=\left\langle x_{1}, x_{2}\right\rangle\right\}$ is $\sigma$-compact for each $x_{i} \in X_{i}$. Then $\left\{\left\langle s_{1}(x), s_{2}(x)\right\rangle \mid x \in X\right\}$ is a measurable subset of $X_{1} \times X_{2}$.

Hence if $s_{i}$ has compact or countable inverse point images, the condition is satisfied, in particular if $s_{i}$ is one-to-one, generalizing the well-known fact that the image of the domain of a one-to-one Borel map on Polish spaces is a Borel set (this generalization is well known, too). If $X_{1}$ and $X_{2}$ are $\sigma$-compact, and both $s_{1}$ and $s_{2}$ are continuous, the condition also applies, thus real-valued functions on a Polish space are captured by Observation 2 .

In what follows, bisimulation will mean 2-bisimulation; we write $O_{1} \sim\langle A, K\rangle$ $\mathrm{O}_{2}$ if $\langle A, K\rangle$ is a bisimulation for $\mathrm{O}_{1}$ and $\mathrm{O}_{2}$. 
Remarks: 1. Desharnais, Edalat and Panangaden [4] define bisimulations between labelled Markov processes as spans of zig-zag morphisms in the category $\mathcal{A}$ of analytic spaces. They work in the full subcategory of $\mathbb{1}_{\mathcal{A}} \downarrow \boldsymbol{S}$ that has objects of the diagonal form $\langle S, S, K\rangle$. Because the product of two analytic spaces is an analytic space again [17, Theorem I.3.2], two labelled Markov processes with probabilistic (rather than sub-probabilistic) transition kernels are always bisimilar.

2. Rutten [18] defines bisimulations through projections: let $\mathcal{S}$ be the category of sets, and $\boldsymbol{F}: \mathcal{S} \rightarrow \mathcal{S}$ a functor, then the pair $\langle S, \alpha\rangle$ with $\alpha: S \rightarrow \boldsymbol{F}(S)$ is a coalgebra, thus coalgebras are diagonal members of a full subcategory of $\mathbb{1}_{\mathcal{S}} \downarrow \boldsymbol{F}$. A bisimulation between the coalgebras $\left\langle S_{1}, \alpha_{1}\right\rangle$ and $\left\langle S_{2}, \alpha_{2}\right\rangle$ is a coalgebra $\langle R, \gamma\rangle$ with $R \subseteq S_{1} \times S_{2}$ such that the projections $\pi_{i}: R \rightarrow S_{i}$ satisfy $\alpha_{1} \circ \pi_{i}=$ $\boldsymbol{F}\left(\pi_{i}\right) \circ \gamma$. In [3], de Rutten and Vink define probabilistic bisimulations through relations quite close to the definition for labelled transition systems given by Milner [14, and the definition given by Larsen and Skou 13. They define also bisimulations for a functor similar to $\boldsymbol{P}$ on diagonal objects. They prove the equivalence on ultrametric spaces for what they call $z$-closed relations with a Borel decomposition ([3], Lemma 5.5, Theorem 5.8).

3. Moss [15, Sect. 3] defines a bisimulation on a coalgebra (rather than for two coalgebras), and he shows that the existence of a bisimulation can be established under rather weak conditions (Prop. 3.10, which he attributes to Aczel and Mendler). Moss works in the category of sets and classes, he bases coalgebras on a functor which is set based, and which preserves weak pullbacks, assumptions that are not met in the situation considered here.

We will show now that bisimulations respect the conventional and the demonic product and start out with a technical observation. It states that bisimilarity is maintained when we restrict the possible inputs (otherwise the similarity of behavior would depend on the chosen base set which would be irritating for a concept which exhibits local properties):

Observation 3 Denote for $K: X \stackrel{\bullet}{\hookrightarrow} Y$ and the measurable subset $\emptyset \neq A \subseteq X$ the restriction of $K$ to $A$ by $K \downarrow A$. Then the following holds:

1. $K \downarrow A: A \stackrel{\bullet}{\hookrightarrow} Y$, and $\langle A, Y, K \downarrow A\rangle$ is a probabilistic object, if $\langle X, Y, K\rangle$ is one.

2. If $\left\langle X_{1}, Y_{1}, K_{1}\right\rangle \sim\langle A, T\rangle\left\langle X_{2}, Y_{2}, K_{2}\right\rangle$, and if $\emptyset \neq A_{i} \subseteq X_{i}$ are measurable $(i=$ 1, 2), then

$$
\left\langle A_{1}, Y_{1}, K_{1} \downarrow A_{1}\right\rangle \sim\langle B, R\rangle\left\langle A_{2}, Y_{2}, K_{2} \downarrow A_{2}\right\rangle,
$$

where $B:=A \cap\left(A_{1} \times A_{2}\right)$, and $R:=T \downarrow\left(A_{1} \times A_{2}\right)$.

We show first that bisimilarity respects the conventional product. Suppose that $\langle A, T\rangle$ and $\langle B, S\rangle$ are the intermediate objects from which the respective spans are obtained. For maintaining bisimilarity, the composition of the mediating objects $\langle A, B, T\rangle$ should be a probabilistic one. This is intuitively clear: the mediating computation should terminate, otherwise similar behavior cannot be carried through the composition. 
We will see subsequently that a mediating probabilistic object is also required for making bisimulation respect demonic products, too.

Proposition 6. Let $K_{i}: X_{i} \stackrel{\bullet}{\hookrightarrow} Y_{i}$, and $L_{i}: Y_{i} \stackrel{\bullet}{\hookrightarrow} Z_{i}(i=1,2)$, and assume that

1. $\left\langle X_{1}, Y_{1}, K_{1}\right\rangle \sim\langle A, T\rangle\left\langle X_{2}, Y_{2}, K_{2}\right\rangle$,

2. $\left\langle Y_{1}, Z_{1}, L_{1}\right\rangle \sim\langle B, S\rangle\left\langle Y_{2}, Z_{2}, L_{2}\right\rangle$.

Then $\left\langle X_{1}, Z_{1}, K_{1} ; L_{1}\right\rangle \sim\langle A, T ; S\rangle\left\langle X_{2}, Z_{2}, K_{2} ; L_{2}\right\rangle$, provided $\langle A, B, T\rangle$ is a probabilistic object.

This carries readily over to the demonic product:

Corollary 3. Put under the assumptions of Proposition [6

$$
C:=A \cap\left(W\left(K_{1}, L_{1}\right) \times W\left(K_{2}, L_{2}\right)\right) \text {, and } R:=(T ; S) \downarrow C,
$$

and assume that $\langle C, B, R\rangle$ is a probabilistic object. Then

$$
\left\langle X_{1}, Z_{1}, K_{1} \star L_{1}\right\rangle \sim\langle C, R\rangle\left\langle X_{2}, Z_{2}, K_{2} \star L_{2}\right\rangle
$$

holds.

\section{Comparing Demonic Products}

A transition kernel $K: X \stackrel{\bullet}{\hookrightarrow} Y$ induces a closed-valued relation via the support function, whenever $Y$ is Polish (see Sect. 2). This correspondence was investigated in [6] with a view towards stochastic and non-deterministic automata, in particular it could be shown under which conditions a closed-valued relation in representable by a Markov kernel. The present line of investigation, however, requires a somewhat more discriminating instrument than the support function, because the set $\operatorname{supp}(K(x))$ does not tell us anything about termination upon input $x$ - it merely states what outputs are produced.

Thus we work with the modified relation

Definition 3. The non-deterministic fringe of the transition kernel $K$ is defined as the relation $\operatorname{supp}^{*} K:=\Delta_{\operatorname{Tr}(K)}$ ○ supp $K$.

Hence the fringe captures exactly those inputs that contribute to termination, composition with relation $\Delta_{\operatorname{Tr}(K)}$ serving as a filter. Thus $\langle x, y\rangle \in \operatorname{supp}^{*} K$ holds iff $K(x)(Y)=1$, and if $y$ is a possible output to $K(x)$.

We will in this section investigate how the demonic product of transition kernels relates to the demonic product of the fringe as a modified support function, hence we will investigate the question under which conditions $\operatorname{supp}^{*}(K \star L)=$ $\operatorname{supp}^{*} K \diamond \operatorname{supp}^{*} L$ (or possibly a weaker subset relation) holds, where $L: X \stackrel{\bullet}{\hookrightarrow} Y$ is the factor to $K$ in this game. This will shed some light on the relationship between the set theoretic and the probabilistic version of the demonic product. 
Since the latter is modelled after the former, it would intuitively be gratifying to see that they behave similar, provided they can be compared at all. The fringe relation serves exactly this purpose by converting a probabilistic relation into a set theoretic one.

Example 4. Let $K$ and $L$ be defined as in Example 2, then

$$
\begin{aligned}
\operatorname{supp}^{*} K & =\{\langle 2, a\rangle,\langle 2, b\rangle,\langle 2, c\rangle,\langle 3, a\rangle,\langle 3, c\rangle\}, \\
\operatorname{supp}^{*} L & =\{\langle a, \alpha\rangle,\langle a, \beta\rangle,\langle a, \gamma\rangle,\langle b, \alpha\rangle,\langle b, \beta\rangle,\langle b, \gamma\rangle\},
\end{aligned}
$$

with

$$
\operatorname{supp}^{*} K \diamond \operatorname{supp}^{*} L=\{\langle 3, \alpha\rangle,\langle 3, \beta\rangle,\langle 3, \gamma\rangle\}=\operatorname{supp}^{*}(K \star L)
$$

(cp. relations $R$ and $S$ from Example 1).

We fix for the rest of this section the measurable space $X$ and the Polish spaces $Y, Z$ as well as the transition kernels $K: X \stackrel{\bullet}{\hookrightarrow} Y$ and $L: Y \stackrel{\bullet}{\hookrightarrow} Z$. L may be assumed to be (weakly) continuous in view of Prop. 2. To avoid trivialities, we assume that both $\operatorname{Tr}(K) \neq \emptyset$ and $\operatorname{Tr}(L) \neq \emptyset$ holds.

It is known from [7, Obs. 4] that the support function has these properties when related to the product of transition kernels:

1. supp $K \circ \operatorname{supp} L \subseteq \operatorname{supp}(K ; L)$,

2. $\operatorname{supp}(K ; L) \subseteq \operatorname{supp} K \circ \operatorname{supp} L$, provided $K(x)(G)>0$ holds for each $x \in X$ and each open ball $G$ in $Y$.

These properties have been established for Markov kernels, but the proofs carry over easily to the present situation.

We collect some readily established properties for the fringe relation:

Observation 4 1. $\operatorname{Tr}(K \star L)=W(K, L)$,

2. $\operatorname{supp}(K \star L)=\Delta_{W(K, L)} \circ \operatorname{supp}(K ; L)=\operatorname{supp}^{*}(K \star L)$,

3. $W(K, L) \times Z=\overline{\operatorname{supp}^{*} K \circ \overline{\operatorname{supp}^{*} L \circ U_{Z}}}$,

4. $\Delta_{W(K, L)} \circ \operatorname{supp} K \circ \operatorname{supp} L \subseteq \operatorname{supp}^{*} K \circ \operatorname{supp}^{*} L$.

With these preparations we are able to characterize the relationship between the demonic product of the fringes, and the fringe of the demonic product:

Proposition 7. The non-deterministic fringe of the demonic product of probabilistic relations and the demonic product of their fringes are related in the following way:

1. $\operatorname{supp}^{*} K \diamond \operatorname{supp}^{*} L \subseteq \operatorname{supp}^{*}(K \star L)$,

2. if supp $(K ; L)=\operatorname{supp} K \circ \operatorname{supp} L$ can be established, then this implies $\operatorname{supp}^{*} K \diamond \operatorname{supp}^{*} L=\operatorname{supp}^{*}(K \star L)$.

Corollary 4. Assume that $K(x)(G)>0$ for each $x \in X$ and each open ball $G$ in $Y$, then supp* $K \diamond \operatorname{supp}^{*} L=\operatorname{supp}^{*}(K \star L)$ holds. 
Suppose that our probabilistic demon $\Pi$ has a little brother $\Sigma$ who is keen on set theoretic relations, and, being junior to $\Pi$, is handed always the fringe. $\Pi$ performs two computations through the demonic product, and hands to $\Sigma$ his share. In general, $\Sigma$ is better off (in the sense of having obtained a larger relation) in not performing the demonic product himself (hence it pays for $\Sigma$ to wait for $\Pi$ doing the combined computation). If the first computation, however, is thrifty by never vanishing on non-empty open sets, then it is of no concern to $\Sigma$ who combines the computations.

\section{Conclusion}

We make in this paper a proposal for the definition of the demonic product of two transition kernels. This product indicates how a probabilistic demon might act when composing two computations that are modelled through probabilistic relations. It turns out that the demonic product coincides with the ordinary product, provided Markov kernels are involved, i.e. computations that terminate with probability one. It could be shown that the demonic product is stable under bisimulations under the provision that the relating object is a Markov kernel. Bisimulations have been defined for the situation at hand, slightly generalizing the notion of bisimulation for stochastic systems given in [4], and relating it to the definition given in the context of coalgebras in [18. The paper shows then that there is a close relationship between the demonic product of two probabilistic relations and the demonic product of their respective fringe relations, the latter product being the set theoretic product, as introduced and investigated in e.g. 5 .

Further investigations in the area of probabilistic relations will deal with bisimulations along the lines suggested by Moss [15], cp. [4], in particular the characterization theorems for bisimulations that have been formulated for coalgebras should be transported to the comma category underlying the discussions here. Less abstract, we will see how bisimulation relates to the converse of a probabilistic relation, as defined and investigated in 7 . We still do not know under which conditions 2-bisimilarity is a transitive relation. In [9] a theory of hierarchical refinement of probabilistic relations is proposed in terms of the usual product. It would be interesting to see what happens when the demonic product is used instead.

\section{References}

1. S. Abramsky, R. Blute, and P. Panangaden. Nuclear and trace ideal in tensored *categories. Technical report, School of Computer Science, McGill University, Montreal, June 1998.

2. C. Brink, W. Kahl, and G. Schmidt, editors. Relational Methods in Computer Science. Advances in Computing Science. Springer-Verlag, Wien, New York, 1997.

3. E. P. de Vink and J. J. M. M. Rutten. Bisimulation for probabilistic transition systems: a coalgebraic approach. Technical Report SEN-R9825, CWI, Amsterdam, 1998. 
4. J. Desharnais, A. Edalat, and P. Panangaden. Bisimulation of labelled markovprocesses. Technical report, School of Computer Science, McGill University, Montreal, 1998.

5. J. Desharnais, A. Mili, and T. T. Nguyen. Refinement and demonic semantics. In [2], pages $166-184$.

6. E.-E. Doberkat. Stochastic Automata - Nondeterminism, Stability, and Prediction, volume 113 of Lecture Notes in Computer Science. Springer-Verlag, Berlin, 1981.

7. E. E. Doberkat. The converse of a probabilistic relation. Technical Report 113, Chair for Software Technology, University of Dortmund, June 2001.

8. E.-E. Doberkat. The demonic product of probabilistic relations. Technical Report 116, Chair for Software Technology, University of Dortmund, September 2001. Available as Technische-Berichte/Doberkat_SWT-Memo-116.ps.gz in directory ftp://1s10-www.cs.uni-dortmund.de/pub/.

9. E.-E. Doberkat. The hierarchical refinement of probabilistic relations. Technical Report 118, Chair for Software Technology, University of Dortmund, November 2001.

10. M. Giry. A categorical approach to probability theory. In Categorical Aspects of Topology and Analysis, volume 915 of Lecture Notes in Mathematics, pages 68 85, Berlin, 1981. Springer-Verlag.

11. C. J. Himmelberg. Measurable relations. Fund. Math., 87:53 - 72, 1975.

12. S. Mac Lane. Categories for the Working Mathematician. Number 5 in Graduate Texts in Mathematics. Springer-Verlag, Berlin, 2 edition, 1997.

13. K. G. Larsen and A. Skou. Bisimulation through probabilistic testing. Information and Computation, 94:1 - 28, 1991.

14. R. Milner. A Calculus of Communicating Systems. Number 92 in Lecture Notes in Computer Science. Springer-Verlag, Berlin, 1980.

15. L. S. Moss. Coalgebraic logic. Annals of Pure and Applied Logic, 96:277 - 317, 1999.

16. P. Panangaden. Probabilistic relations. In C. Baier, M. Huth, M. Kwiatkowska, and M. Ryan, editors, Proc. PROBMIV, pages $59-74,1998$. Also available from the School of Computer Science, McGill University, Montreal.

17. K. R. Parthasarathy. Probability Measures on Metric Spaces. Academic Press, New York, 1967.

18. J. J. M. M. Rutten. Universal coalgebra: a theory of systems. Technical Report CS-R9652, CWI, Amsterdam, 1996.

19. S. M. Srivastava. A Course on Borel Sets. Number 180 in Graduate Texts in Mathematics. Springer-Verlag, Berlin, 1998. 